\title{
AN EQUIVARIANT TORUS THEOREM FOR INVOLUTIONS
}

\author{
W. H. HOLZMANN
}

\begin{abstract}
A complete classification is given for equivariant surgery on incompressible tori with respect to involutions with possible 1- or 2-dimensional fixed sets.
\end{abstract}

\section{INTRODUCTION}

In [8] an equivariant torus theorem was proved for involutions which have at most isolated fixed points. The main result of this paper is an equivariant torus theorem (Theorem 4.5) for involutions with possible 1- or 2-dimensional fixed sets. If additional restrictions are imposed, various equivariant surgery theorems have been proved. If the manifold is closed, orientable, and irreducible with infinite first homology, a theorem was given in [12].

The proof of the equivariant torus theorem proceeds by isotoping a given incompressible torus to a new torus such that the new torus, its image under the involution, and the fixed point set of the involution are "almost pairwise transversal." The weaker "almost pairwise transversality" condition is used since, in general, pairwise transversality cannot be ensured (see Remark 3.3). The curves of intersection between the new torus and its image under the involution are of certain types. The curves are changed or removed by various surgeries, thus obtaining an equivariant torus or one of the exceptional cases as listed in the theorem.

An application of this theorem is the classification of involutions with 1- or 2-dimensional fixed sets on orientable torus bundles or unions of twisted $I$ bundles on Klein bottles. Surgery on an equivariant torus reduces these spaces to spaces on which the involutions are known. Details of this application are given in [4].

\section{Preliminaries}

Throughout we use the piecewise linear category. A piecewise linear homeomorphism will be called an isomorphism.

Received by the editors January 20, 1988 and, in revised form, February 1, 1990.

1980 Mathematics Subject Classification (1985 Revision). Primary 57A10, 57E25.

Key words and phrases. Involution, fixed sets, equivariant surgery.

The author wishes to thank the referee for his comments and suggestions. 
Let $M$ be a connected compact 3-manifold. An involution $l$ is an isomorphism with $l \neq$ id and $l^{2}=\mathrm{id}$.

Let Fix denote the fixed set Fix $=\operatorname{Fix}(l)=\{x: l(x)=x\}$. Let $l$ be an involution on a manifold $M$ and $l^{\prime}$ an involution on a manifold $M^{\prime} . l$ and $l^{\prime}$ are conjugate if there is an isomorphism $h: M \rightarrow M^{\prime}$ with $l^{\prime}=h \circ l \circ h^{-1}$. Call $h$ a conjugation between $l$ and $l$.

Lemma 2.1. Given a simplicial subdivision $K$ of $M$ and an involution $l$ of $M$ there is a subdivision $L$ of $K$ with $l$ simplicial with respect to $L$.

Proposition 2.2. Let $l$ be an involution on a manifold $M$. Let $L$ be a subdivision of $M$ with $l: L \rightarrow L$ simplicial, and let $L^{\prime}$ be the first barycentric subdivision of $L$. Then Fix $=\operatorname{Fix}(l)$ is a subcomplex of $L^{\prime}$. Fix is the union of disjoint 0-, 1- and 2-dimensional proper submanifolds. Write $\mathrm{Fix}^{0}, \mathrm{Fix}^{1}$, and $\mathrm{Fix}^{2}$ respectively for the unions of the 0-, 1- and 2-dimensional components of Fix.

If $v \in \mathrm{Fix}^{0} \cup \mathrm{Fix}^{2}$ then $\mathrm{l}$ is locally orientation reversing at $v$. If $v \in \mathrm{Fix}^{1}$ then $l$ is locally orientation preserving at $v$. In particular, if $M$ is orientable then $\mathrm{l}$ is orientation reversing if $\mathrm{Fix}^{0} \cup \mathrm{Fix}^{2} \neq \varnothing$ and $l$ is orientation preserving if $\operatorname{Fix}^{1} \neq \varnothing$.

Proof. Use the following:

(1) Let $\Delta$ be a standard $m$-simplex (with standard subdivision) invariant under $l$. Then Fix $\cap \Delta$ is a subcomplex of the first barycentric subdivision of $\Delta$.

(2) If Fix contains a 3-simplex then $l=\mathrm{id}$.

If $v \in$ Fix is a vertex of $\operatorname{int}(L)$ consider the link $L k$ of $v$.

(3) If $L k \cap$ Fix contains a 1-cell then $L k \cap$ Fix is one 1-sphere. So $v \in \mathrm{Fix}^{2}$.

(4) If $L k \cap$ Fix consists of $m \geq 0$ vertices then

$$
\chi(L k / \imath)-m=\frac{1}{2}(\chi(L k)-m) .
$$

Since $L k / l$ is a surface and $L k$ is a 2 -sphere, it follows that $m=2$ and hence $v \in \mathrm{Fix}^{1}$, or $m=0$ and hence $v \in \mathrm{Fix}^{0}$.

\section{EQUIVARIANT TRANSVERSALITY}

In order to be able to perform surgeries on a surface $F_{0}$ in a 3-manifold $M$ we would like to perform an ambient isotopy on $F_{0}$ such that the isotopic surface $F$ has the property that $F, l F$, and Fix are pairwise transversal. This can be done if the manifold is orientable. If Fix $^{2} \neq \varnothing$ and $M$ is nonorientable, however, pairwise transversality is not possible in general. This necessitates using a somewhat weaker form of transversality.

Lemma 3.1. Let $F$ be a proper surface in a 3-manifold with $F, \imath F$, and Fix pairwise transversal. Then the components of $F \cap \imath F$ are 1-spheres and proper 1-cells. If $C$ is a component of $F \cap l F$ with $C \cap \mathrm{Fix}^{2} \neq \varnothing$ then $C \subseteq \mathrm{Fix}^{2}$. 


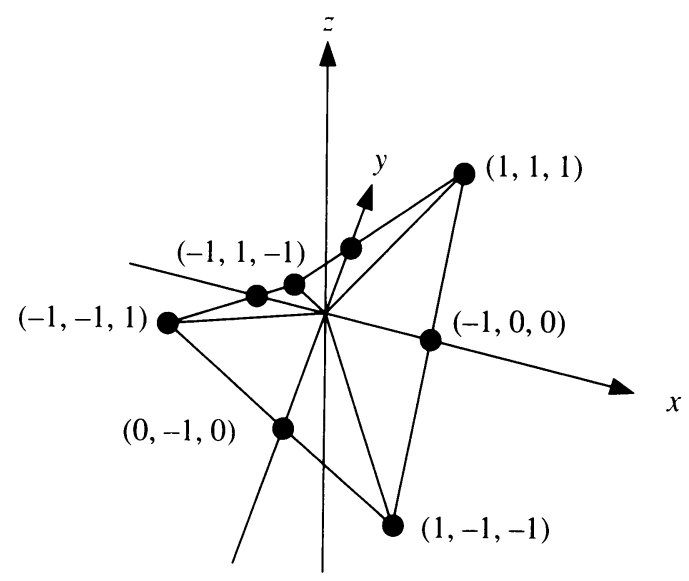

FIGURE 1

Proof. The first statement follows by transversality of $F$ and $l F$. The second statement follows by considering the star of a point in $C \cap \mathrm{Fix}^{2}$.

For the 3-cell $B^{3}=\{(x, y, z):|x| \leq 1,|y| \leq 1,|z| \leq 1\}$ in $\mathbf{R}^{3}$ let $i: B^{3} \rightarrow B^{3}$ be the map $i(x, y, z)=(-x, y, z)$. Then $\operatorname{Fix}(i)$ is the intersection of $B^{3}$ with the $y z$ plane. Let $S$ be the 1-sphere obtained as the join of $\{(1,1,1),(-1,-1,1)\}$ with $\{(-1,1,-1),(1,-1,-1)\}$ and let $D$ be the cone from $(0,0,0)$ on $S . D$ is a saddle shaped region (see Figure 1). (We could alternately take $D$ defined by $\left\{z=x y / \sqrt{x^{2}+y^{2}}\right\} \cup\{(0,0,0)\}$.) Notice that $D \cap i D$ is the part of the $x$ and $y$ axis in $B^{3}$ while $D \cap \operatorname{Fix}(i)$ is part of the $y$ axis. $D$ and $\operatorname{Fix}(i)$ are transversal and $i D$ and $\operatorname{Fix}(i)$ are transversal, but $D$ and $i D$ are not transversal at $(0,0,0)$. There is a subdivision making these spaces simplicial with all the vertices on $\partial B^{3} \cup(0,0,0)$.

Definition 3.2. Let $F$ be a proper surface in a 3-manifold $M$ and $l$ an involution on $M$ with fixed set Fix. Call a point $v$ a saddle point if $v \in F \cap \mathrm{Fix}^{2}$ and if $(F, \imath F, \operatorname{Fix}) \cap \operatorname{star}(v)$ is isomorphic to $(D, i D, \operatorname{Fix}(i))$.

Remark 3.3. Saddle points exist since $i$ is an involution with fixed set $\operatorname{Fix}(i)$. Although $\partial D, \partial i D, \partial \mathrm{Fix}(i)$ are pairwise transversal there is no 2-cell $E$ with $\partial E=\partial D$ and $E, i E, \operatorname{Fix}(i)$ pairwise transversal. Otherwise, since $\partial E \cap$ $\partial i E-\operatorname{Fix}(i)=( \pm 1,0,0)$ there is a $\hat{i}$-cell $I$ of $E \cap i E$ with $(1,0,0) \in \partial I$ and this 1-cell must meet Fix, contradicting the previous lemma.

Let $d$ denote the identification $(x, 1, z) \sim(x,-1,-z)$ for all $x$ and $z$. Then $D / d$ is an annulus in a solid Klein bottle $B^{3} / d$ and no isotopy of $D / d$ moves it to an annulus with $F, i F$, and $\mathrm{Fix}(i) / d$ pairwise transversal.

Definition 3.4. Let $F$ be a proper surface in a 3-manifold and $l$ an involution on $M$ with fixed set Fix. Then $F, \imath F$, and Fix are almost pairwise transversal 
if:

(1) $F, l F$, and Fix are pairwise transversal except at a finite number of saddle points, and

(2) the only components of $F \cap$ Fix containing saddle points are 1-spheres and each such 1-sphere contains at most one saddle point.

Let $\mathscr{E}$ be the closure of $\left(F \cap_{l} F\right)-$ Fix $^{2} . \mathscr{E}$ consists of disjoint 1 -spheres and proper 1-cells: in a neighborhood of a saddle point, $F \cap \imath F-$ Fix $^{2}$ corresponds to $[-1,0) \times 0 \times 0 \cup(0,1] \times 0 \times 0$ in the $B^{3}$ model for saddle points.

Let $E$ be a component of $\mathscr{E}$ that contains a saddle point $v$. Then $E$ has a fixed point and is invariant under $l$. Therefore, either $E$ is a 1 -cell with no fixed points other than $v$ or $E$ is a 1 -sphere with exactly two fixed points $v$ and $w$. By transversality $w$ is in $\operatorname{Fix}^{1}$ or $\operatorname{Fix}^{2}$. In the latter case $w$ is a saddle point. Together with Lemma 3.1 this shows:

Proposition 3.5. Let $F, l F$, and Fix be almost pairwise transversal. Then the components of $F \cap \imath F$ are of one of the following forms:

(1) Components with no saddle points (standard components):

(a) proper 1-cell $I$ with $I \cap$ Fix $=\varnothing$ or $I \subseteq$ Fix $^{2}$,

(b) proper 1-cell I with $I \cap \mathrm{Fix}=I \cap \mathrm{Fix}^{1}=v, v$ a point,

(c) 1-sphere $S$ with $S \cap$ Fix $=\varnothing$ or $S \subseteq$ Fix $^{2}$,

(d) 1-sphere $S$ with $S \cap \mathrm{Fix}=S \cap \mathrm{Fix}^{1}=v_{1} \cup v_{2}$, where $v_{1}$ and $v_{2}$ are points.

(2) Components with saddle points:

Type I component: $S_{1} \cup I$ with $S_{1} \cap I=$ Fix $\cap I=w, S_{1} \subseteq$ Fix $^{2}$, and $w$ is the only saddle point on $S_{1} \cup I$.

Type II component: $S_{1} \cup S$ with $S_{1} \cap S=w, S_{1} \subseteq$ Fix $^{2}, S \cap$ Fix $=v \cup w$, $v \in \mathrm{Fix}$, and $w$ is the only saddle point on $S_{1} \cup S$.

Type III component: $S_{1} \cup S_{2} \cup S$ with $S_{1} \cap S_{2}=\varnothing, S_{i} \cap S=w_{i}, S_{i} \subseteq$ Fix $^{2}, S \cap \mathrm{Fix}=w_{1} \cup w_{2}$, and $w_{1}$ and $w_{2}$ are the only saddle points on $S_{1} \cup S_{2} \cup S$.

Here $S, S_{1}$, and $S_{2}$ are 1-spheres, the $I$ are 1-cells and $w_{i}$ are points, where $i=1,2$.

Note that case (2) does not arise if the manifold is orientable because a regular neighborhood $N$ of $S_{1}$ is always a solid Klein bottle. For if $N$ is instead a solid torus instead, then $\operatorname{Fix} \cap N, F \cap N$, and $\imath F \cap N$ are all annuli or all Möbius bands. Consider annulus $A$, a component of $\partial N-$ Fix . $A \cap F$ and $A \cap l F$ are two 1 -spheres intersecting transversally in $A$. One sees these 1 -spheres have nonzero intersection number, a contradiction.

Corollary 3.6. If $F, \imath F$, and Fix are almost pairwise transversal, then they are pairwise transversal if one of the following holds:

(a) $M$ is orientable.

(b) $F$ is a 2-cell.

(c) $F$ is an annulus with $\partial F \cap \imath \partial F=\varnothing$. 
Proof. In case (a) regular neighborhoods of 1-spheres are solid tori.

In cases (b) and (c) Type II or III components are excluded since the 1-sphere $S$ is nonseparating. In case (c) Type I components are excluded a priori, while in case (b) the 1-sphere $S_{1}$ separates so a proper 1-cell $C$ cannot intersect $S_{1}$ transversally at one point.

In a surface $F$ a proper 1-cell $I$ bounds a disc $D$ if $I=\overline{\partial D-\partial F}$.

Corollary 3.7. Let $F, l F$, and Fix be almost pairwise transversal and $C$ a proper 1-cell or 1-sphere component of $F \cap \imath F$, that is, let $C$ be a standard component. Then any disc in $F$ or $l_{F}$ bounded by $C$ contains only standard components.

Proof. The proof is similar to the proof of case (b) in the previous corollary.

Equivariant Transversality Theorem 3.8. Let $l$ be an involution on a 3-manifold $M$ with $\operatorname{Fix}=\operatorname{Fix}(l)$ and let $F_{0}$ be a proper surface in $M$. Then there is an ambient $\varepsilon$-isotopy on $M$ taking $F_{0}$ to a proper surface $F$ such that $F, l F$, and Fix are almost pairwise transversal. In $\partial M$, if $\partial F, l \partial F$, and Fix are pairwise transversal then the isotopy may be taken to be the identity on $\partial M-N$, where $N$ is a given neighborhood of $\partial \mathrm{Fix}^{2} \cap \partial F$.

Proof. Let $F=F_{0}$ be a proper surface. By Proposition 2.2 and Lemma 2.1 subdivide $M$ so that $l$ is simplicial with respect to the subdivision, Fix is a subcomplex of the subdivision, and Fix is a disjoint union of $0-, 1-$ and 2dimensional components $\mathrm{Fix}^{0}, \mathrm{Fix}^{1}$, and $\mathrm{Fix}^{2}$. All isotopies performed in the construction will be done in the star neighborhoods of certain simplexes. By taking a sufficiently fine subdivision $\varepsilon$-isotopies are obtained.

Step 1. Adjust $F$ near $\mathrm{Fix}^{2}$. By isotopies similar to those in the third step below we can assume $F$ and Fix are transversal, the isotopy not moving $\partial F$ unless $\partial F$ and $\partial F i x$ are nontransversal. In particular, $F \cap \mathrm{Fix}^{0}=\varnothing$. Then $F \cap \mathrm{Fix}^{2}$ consists of disjoint 1-spheres and 1-cell components proper in $M$.

Let $S$ be a 1-sphere component of $F \cap \mathrm{Fix}^{2}$. Let $N^{\prime}$ be a regular neighborhood of $S$ with $N^{\prime} \cap F$ and $N^{\prime} \cap$ Fix transversal and each an annulus or Möbius band. $S$ has a regular neighborhood $N$ contained in $\operatorname{int}\left(N^{\prime}\right)$ invariant under $l$ with no vertices on $\operatorname{int}(N)-S$ such that $N \cap$ Fix is a regular neighborhood of $S$ and Fix $\cap \partial N$ has a regular neighborhood $Q$ in $\partial N$ which is invariant under $l$ and has no vertices except on Fix $\cup \partial Q$.

Case 1. $F \cap N$ and Fix $\cap N$ are annuli. Then $N$ is a solid torus, $\partial Q$ has four components, and $N$ - Fix consists of two components $N_{1}$ and $N_{2}$ which are interchanged by $l$. Let $J_{1}$ and $J_{2}$ be components of $\partial Q$ with $J_{i} \subseteq N_{i}$ and ${ }_{l} J_{1} \neq J_{2}$. Let $A_{i}$ be the annulus with $\partial A_{i}=J_{i} \cup S$ having no vertices except on $\partial A_{i} . F$ is isotopic to a surface $F^{\prime}$ by an ambient isotopy which is the identity on $M-N^{\prime}$ and such that $F^{\prime} \cap N^{\prime} \cap$ Fix $\subseteq N$ and $F^{\prime} \cap N=A_{1} \cup A_{2}$. Since $\imath J_{1} \neq J_{2}$ it follows $F^{\prime} \cap N \cap \imath\left(F^{\prime} \cap N\right)=S$ and $F^{\prime} \cap N, l\left(F^{\prime} \cap N\right)$, Fix $\cap N$ are pairwise transversal. 
Case 2. $F \cap N$ and Fix $\cap N$ are Möbius bands. Then $N$ is a solid torus, and $\partial Q$ has two components that are interchanged by $l$. If $J$ is one of these, then $J$ and $S$ determine a Möbius band $A$ with $\partial A=J$. Proceed as in Case 1.

If $M$ is orientable then Cases (3) and (4) do not arise. Only in these cases do saddle points arise.

Case 3. $F \cap N$ is an annulus and Fix $\cap N$ is a Möbius band. Then $N$ is a solid Klein bottle. Let $A$ be one of the two (open) Möbius band components of $\partial N-$ Fix. There are two 1 -spheres $J_{1}$ and $J_{2}$ which represent generators of $H_{1}(A)=\mathbf{Z}$ with $J_{1}$ and $J_{2}$ intersecting transversally and at only one point $x$. $J_{i}$ and $S$ bound an annulus $A_{i}$ with $A_{1} \cap A_{2}=S \cup I$, where $I$ is a 1-cell with $\partial I=x \cup y, y \in S$. Proceed as in Case 1 using $F^{\prime} \cap N=A_{1} \cup \imath A_{2}$. Then $y$ is a saddle point and $F^{\prime} \cap N, l\left(F^{\prime} \cap N\right)$, and Fix $\cap N$ intersect pairwise transversally elsewhere in $N$.

Case 4. $F \cap N$ is a Möbius band and $\mathrm{Fix} \cap N$ is an annulus. This case is similar to Case 3. Here $A=\partial N-Q$ is an invariant annulus under $l$. Find a curve $J$ that bounds a Möbius band $B$ with $B \cap \imath B=S$ by lifting (from annulus $A / l$ ) a curve $J^{\prime}$ which represents twice a generator and which is embedded in $A / l$ except for one transversal self-intersection. Now proceed as before.

When $S$ is a 1-cell component of $F \cap F_{i x}{ }^{2}$, use an isotopy similar to the one of Case 1 above. This isotopy may change $\partial F$ in $N \cap \partial M$.

Step 2. Adjust $F$ near $\mathrm{Fix}^{1}$. By Step $1, F \cap \mathrm{Fix}^{1}$ consists of a number of vertices in $\operatorname{int}(M)$. If $v \in F \cap \mathrm{Fix}^{1}$, let $N^{\prime}$ be a regular neighborhood of $v$ and let $N$ be the star neighborhood of $v$. Take the subdivision so that $N$ is in the interior of $N^{\prime}, F \cap N$ is a proper 2-cell in $N$, and Fix $\cap N$ is a proper 1-cell. Since $F$ is transversal, $F \cap \partial N$ is a generator of $H_{1}(N-$ Fix $)$. Let $J^{\prime}$ be a curve in the annulus $(\partial N-$ Fix $) / l$ representing twice a generator of this annulus. Take $J^{\prime}$ embedded except for one transversal self-intersection. $J^{\prime}$ lifts to two 1 -spheres $J$ and $l J$, which on coning to $v$ give 2-cells $D$ and $l D$. $D, l D$, and Fix are pairwise transversal in int $N$. Proceed as in Case 1 of Step 1.

We obtain a surface $F$ and a neighborhood $N$ of Fix such that $F$ has the required transversality properties in $N$. The following construction adjusts $F$ only on star neighborhoods of simplexes of $\overline{F-N}$ where $F$ and $l F$ are not already pairwise transversal. By subdividing sufficiently we may assume without loss that $\mathrm{Fix}=\varnothing$. For convenience assume also $\partial F=\varnothing$.

Let $K$ be a subdivision of $M$ with $l$ simplicial and $F$ a subcomplex of $K$. Let $\Delta$ be an $m$-simplex of $F$ in $K$ with $m=0,1$, or 2 . Define $\operatorname{St}(\Delta)$, the reduced star of $\Delta$ in $K$, to be all 3-simplexes $\sigma$ of $K$ with $\Delta \subseteq \sigma$ together with their faces. Let $\mathrm{St}_{F}(\Delta)$, the reduced star of $\Delta$ in $F$, be all 2-simplexes $\sigma$ of $K$ with $\Delta \subseteq \sigma \subseteq F$ together with their faces. Let $p: M \rightarrow M / l$ be the projection. 
Step 3. There is a subdivision of $M$ and a proper surface $F^{\prime} \varepsilon$-isotopic to $F$ such that for every simplex $\Delta$ of $F^{\prime}$ either $p^{-1} p(\Delta) \cap F^{\prime}=\Delta$ or $\Delta$ is a 0 - or 1-simplex with $\operatorname{int}\left(\mathrm{St}_{F^{\prime}}(\Delta)\right)$ and $\operatorname{int}\left(\mathrm{St}_{l F^{\prime}}(\Delta)\right)$ transversal.

Call a simplex exceptional if it fails to satisfy these conditions and is of the highest possible dimension $m=0,1$, or 2 . Induct on the number of such simplexes. If there are no exceptional simplexes the theorem is established.

Suppose there is an exceptional simplex $\Delta$. Adjoin all the vertices (and their translates under $l$ ) of form $\frac{m+2}{m+3} b+\frac{1}{m+3} v$, where $b$ is the barycenter of $\Delta$ and $v$ is a vertex of $\operatorname{St}(\Delta)-\Delta$. This determines a refinement $K^{\prime}$ of $K$ with the same number of exceptional simplexes; no $m$-simplexes are subdivided for $m=1,2$, while for $m=0$ transversality already holds away from vertices of $K$. Consider the reduced stars in $K^{\prime} . \partial \mathrm{St}_{F}^{\prime}(\Delta)$ is a 1 -sphere that decomposes $\partial \mathrm{St}^{\prime}(\Delta)$ into two components $D_{+}$and $D_{-}$. There is an ambient isotopy taking $F$ to $F_{1}=\left(F-\mathrm{St}_{F}^{\prime}(\Delta)\right) \cup D_{+}$which is the identity except on $\operatorname{St}_{F}^{\prime}(\Delta) . F_{1}$ has fewer exceptional simplexes. When $m \neq 2$ this follows since $D_{+} \cup D_{-}$intersects the interior of any 2 -simplex of $\operatorname{St}(\Delta)$ transversally.

Regular neighborhoods of the standard components of $F \cap l F$ can be taken in a special form.

Proposition 3.9. Let $F, l F$, and Fix be almost pairwise transversal and $S$ be a 1-sphere component of $F \cap l F$. Suppose, in addition, that the regular neighborhood of $S$ in $F$ and $I F$ is an annulus. Then there exists a regular neighborhood $V \subseteq \operatorname{int}(M)$ of $S$, called a standard neighborhood of $S$, with the following properties:

(1) $V \cap F$ and $V \cap \imath F$ are annuli. Since these intersect transversally, $V$ is a solid torus.

(2) Fix and $\partial V$ intersect transversally, Fix $\cap F \cap V \subseteq S$, and the closure of each component of $(\mathrm{Fix} \cap V)-S$ meets $S$ and $\partial V$. In particular, $\operatorname{Fix}^{0} \cap V=\varnothing$.

(3) Fix $\cap V$ is an annulus, two proper 1-cells, or empty.

(4) If $\imath S=S$ then $\imath V=V$.

(5) If $\imath S \neq S$ then $\imath V \cap V=\varnothing$ and the above properties hold simultaneously for $l V$.

Property (3) can be arranged since if Fix $\cap S \neq \varnothing$ then $l S=S . \quad l$ is an involution on a 1 -sphere so either $l=$ id or $l$ has exactly two fixed points.

The four 1-spheres $(F \cup \imath F) \cap \partial V$ decompose $\partial V$ into four (closed) annuli $\alpha_{1}, \alpha_{2}, \beta_{1}$, and $\beta_{2}$ with $\alpha_{1} \cap \alpha_{2}=\varnothing$ and $\beta_{1} \cap \beta_{2}=\varnothing$. Call these annuli the standard annuli corresponding to the standard neighborhood of $V$. Suppose $l S=S$. Relabeling, if necessary, we may assume $l\left(\alpha_{1} \cap \beta_{1}\right)=\left(\alpha_{1} \cap \beta_{2}\right)$. It follows that $l \alpha_{1}=\alpha_{1}$. Then $l \beta_{1}=\beta_{2}$ and $l \alpha_{2}=\alpha_{2}$. When Fix $\cap V \neq \varnothing$ we obtain Fix $\cap \alpha_{1} \neq \varnothing$, Fix $\cap \alpha_{2} \neq \varnothing$, Fix $\cap \beta_{1}=\varnothing=$ Fix $\cap \beta_{2}$, and each component of Fix $\cap V$ meets both $\alpha_{1}$ and $\alpha_{2}$.

Proposition 3.10. Let $S$ be a 1-cell component of $F \cap \imath F$, where $F, \imath F$, Fix are pairwise transversal (near $S$ ). Then there exists a regular neighborhood $V$ of 
$S$ with $V \cap \partial M$ a regular neighborhood of $\partial S$, called a standard neighborhood of $S$, with the following properties:

(1) $V \cap F$ and $V \cap l F$ are 2-cells with $\partial M \cap V \cap F$ and $\partial M \cap V \cap \imath F$ each two 1-cells. Necessarily $V$ is a 3-cell.

(2), (4) and (5) as for 1-sphere standard neighborhoods.

(3) Fix $\cap V$ is a disc, one proper 1-cell, or empty.

The four 1-cells $(F \cup l F) \cap \overline{\partial V-\partial M}$ subdivide $\overline{\partial V-\partial M}$ into four discs $\alpha_{1}, \alpha_{2}, \beta_{1}$, and $\beta_{2}$ with $\alpha_{1} \cap \alpha_{2}=\varnothing, \beta_{1} \cap \beta_{2}=\varnothing$, and the properties as in the previous situation. Call these discs the standard discs corresponding to $V$.

Remark 3.11. In the following theorem certain 1-sphere components $S$ of $F \cap$ $\imath F$ have standard neighborhoods because $S$ bounds discs in $F$ and $\imath F$. In the disc theorem $F$ is orientable so again there are standard neighborhoods. In the torus theorem the construction will be made so as to keep $S$ in this form always.

Theorem 3.12. Let $M$ be an irreducible 3-manifold with involution $l$ and $F_{0}$ be an incompressible proper surface. Then there is an ambient isotopy of $M$ which is an E-isotopy on $\partial M$ taking $F_{0}$ to a proper surface $F$ such that $F$, $\imath F$, and Fix are almost pairwise transversal and no 1-spheres in $F \cap \imath F$ bound 2-cells in $F$. On $\partial M$, if $\partial F, \imath \partial F$, and $\partial F i x$ are pairwise transversal then the isotopy may be taken to be the identity on $\partial M-N$, where $N$ is a given neighborhood of $\partial \mathrm{Fix}^{2} \cap \partial F$.

Proof. By the preceding transversality theorem there is an $F$ with all the above properties except possibly 1-spheres in $F \cap l F$ bound 2-cells in $F$. By Proposition 3.5 those 2-cells contain no saddle components. Let $S$ be a 1-sphere of $F \cap \imath F$ bounding an innermost 2-cell in $\imath F$, that is, there is a 2-cell $D \subseteq \imath F$ with $D \cap F=\partial D=S$. Since $F$ is compressible, $S$ bounds a 2-cell $B$ in $F$. If $l S=S$ then we may assume $\imath B=D$.

Let $V$ be a standard neighborhood of $S$. Such a neighborhood exists since $S$ bounds a disc in $F$ and $l F$. Let $\alpha$ be the standard annulus in $\partial V$ meeting $D$ but not $B$. Then $l \alpha \cap \alpha=\varnothing$. There is a bicollar $D \times[-1,1]$ of $D=D \times 0$ with

$$
\partial D \times[-1,1]=D \times[-1,1] \cap F=S \times[-1,1]
$$

and with $D \times 1 \cap \alpha \neq \varnothing$. Since $D$ is innermost it follows that for a sufficiently thin collar $(D \times 1) \cap l(D \times 1)=\varnothing$ and $F \cap l(D \times 1)=\varnothing$. Consider $F^{\prime}=$ $(F-(B \cup S \times[-1,1])) \cup D \times 1$. Then $F^{\prime} \cap \imath F^{\prime} \subseteq(F \cap \imath F)-S$ and $F^{\prime}, \imath F^{\prime}$, and Fix are almost pairwise transversal. Since $M$ is irreducible and $D \cup B$ is a 2-sphere, $F^{\prime}$ and $F$ are ambient isotopic by an isotopy being the identity on $\partial M$. By induction, all 1-spheres bounding 2-cells can be removed.

A 2-cell $B$ in a 3-manifold is essential if it is proper and $\partial B$ does not bound a 2-cell in $\partial M$. In an irreducible 3-manifold a nonseparating proper 2-cell is essential. The following theorem is well known (see [1, 2, 6, or 10] for instance). 
Disc Theorem 3.13. Let $M$ be an irreducible 3-manifold with involution $l$. Suppose $M$ has an essential 2-cell $B_{0}$. Then there is an essential 2-cell $B \subseteq M$ such that $B$ and Fix are transversal and either $B \cap l B=\varnothing$ or $l B=B$. In the former case $B \cap \mathrm{Fix}=\varnothing$ and in the latter case $B \cap \mathrm{Fix}$ is a proper 1-cell of $B$ or one point in the interior of $B$. If $\partial B_{0} \cap \imath \partial B_{0}=\varnothing$ then one can take $\partial B=\partial B_{0}$ and $B$ and $B_{0}$ are ambient isotopic by an isotopy that is the identity on $\partial M$.

Proof. By Theorem 3.12 and Corollary 3.6 there is an essential 2-cell $B$ with $B, l B$, and Fix pairwise transversal, $B$ and $B_{0}$ ambient isotopic, and $B \cap l B$ is either empty or consists of proper 1-cells only. Assume $B \cap l B \neq \varnothing$ (in particular, then $\left.\partial B_{0} \cap \imath \partial B_{0} \neq \varnothing\right)$. By induction it suffices to show how to obtain a new 2-cell $B_{i}$ with fewer 1-cells in $B_{i} \cap \imath B_{i}$.

Let $D$ be an outermost disc of $B: D \subseteq B$ with $D \cap \imath B=\partial D \cap \imath B=I$ a proper 1-cell of $B$ and $\partial D-I \subseteq \partial B$. If $l I=I$ define $D^{\prime}=\overline{l B-l D}$. If $l I \neq I$ define $D^{\prime}$ to be the closure of the component of $l B-I$ that does not contain $l I$ (see Figure 2). Let $V$ be a standard neighborhood of $I$ and let $\alpha_{1}$, $\alpha_{2}$, and $\beta$ be standard discs of $V$ with $\alpha_{1} \cap \alpha_{2}=\varnothing, \alpha_{1} \cap \beta \cap D \neq \varnothing$, and $\beta \cap D^{\prime} \neq \varnothing$. Consider

$$
B_{1}=\left(D \cup \beta \cup D^{\prime}\right)-\operatorname{int}(V) \text { and } B_{2}=D \cup\left(l B-D^{\prime}\right) .
$$

Then $B_{1} \cap \imath B_{1} \subseteq(B \cap \imath B)-I$. If $B_{1}$ is essential we are done by induction or we arrive at the case $B_{1} \cap \imath B_{1}=\varnothing$. If $B_{1}$ is not essential then $\partial B_{1}$ bounds a 2-cell $E$ of $\partial M$. Since $M$ is irreducible, the 2-sphere $B_{1} \cup E$ bounds a 3-cell. This 3-cell does not meet $I$, otherwise $l B$ would not be essential. Using the 3-cell construct an ambient isotopy taking $B_{2}$ to $l B$.

So we may assume $B_{2}$ is essential. If $I=l I$ we have $l B_{2}=B_{2}$ and note that Fix $\cap B_{2} \subseteq$ Fix $\cap I$, which is necessarily a point of $I$ or all of $I$. If $I \cap I=\varnothing$ consider a sufficiently thin bicollar $D \times[-1,1]$ of $D=D \times 0$ such that $D \times[-1,1] \cap I$ is a bicollar of $I$ in $l B$ and $D \times 1$ meets $\alpha_{1}$. Then $B_{2}^{\prime}=(D \times 1 \cup l B)-\left(I \times[-1,1) \cup D^{\prime}\right)$ is essential since it is isotopic to $B_{2}$ and $B_{2}^{\prime} \cap \imath B_{2}^{\prime} \subseteq B \cap \imath B-I$.
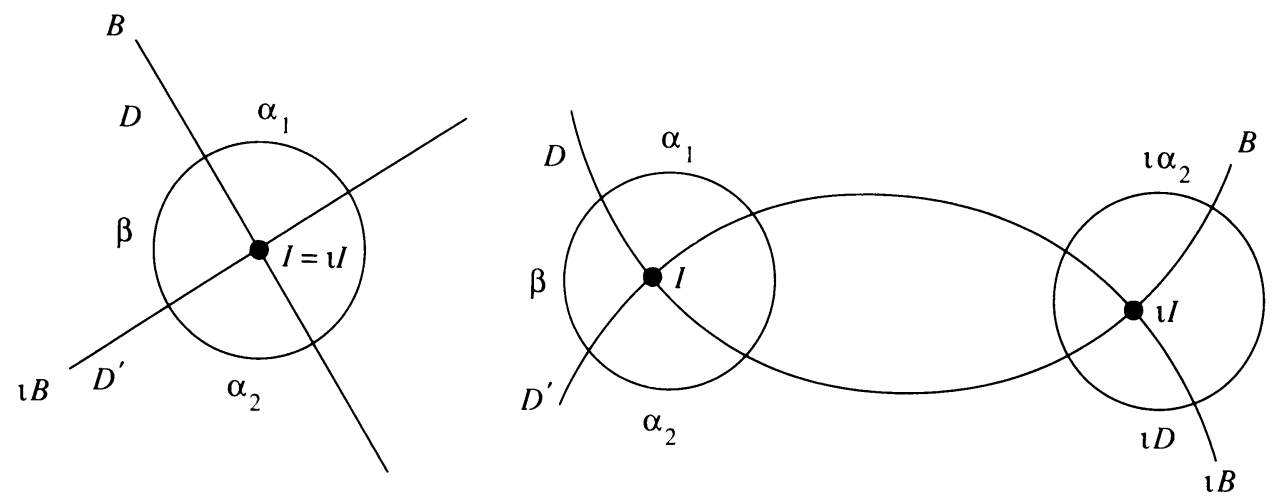

FIGURE 2 


\section{EQUIVARIANT TORUS THEOREM}

A proper annulus $A$ in a 3-manifold $M$ is trivial if $A$ decomposes $M$ into a solid torus $V=D^{2} \times S^{1}$ and a submanifold $M_{0}$ such that:

$$
M=M_{0} \cup V, \quad M_{0} \cap V=\partial M_{0} \cap \partial V=A,
$$

and there exists a nonseparating proper 2-cell $B \subseteq V$ with $B \cap A=\partial B \cap A$ a nonseparating 1-cell in $A$.

Otherwise call $A$ nontrivial. Call $V$ a solid torus that trivializes $A$.

Note that if $A$ does not separate $M$ or if $\partial A$ is in different boundary components of $M$ then $A$ is nontrivial.

Call a nontrivial incompressible proper annulus an essential annulus. Call an incompressible proper Möbius band an essential Möbius band.

Let $F$ be a surface and $S$ a component of $F \cap \imath F$. In some surgeries performed later we will wish to replace $F$ by $F^{\prime}=F \times 1$, where $F \times[-1,1]$ is a bicollar of $F$. To insure that $F^{\prime}, l F^{\prime}$, and Fix are transversal at least in standard neighborhoods (see Propositions 3.9 and 3.10), the following lemma is used.

Lemma 4.1. Let $V$ be a solid torus and $l: V \rightarrow V$ an involution. Let $A_{0}$ and $A_{1}$ be annuli in $\partial V$ with $\partial A_{0}=S_{0} \cup S_{1}, \partial A_{1}=S_{1} \cup S_{2}, A_{0} \cap A_{1}=S_{1}$, $\imath S_{0}=S_{0},\left(A_{0} \cup A_{1}\right) \cap \imath\left(A_{0} \cup A_{1}\right)=S_{0}$, and Fix $\cap\left(A_{0} \cup A_{1}\right) \subseteq S_{0}$. Then there is a proper annulus $A \subseteq V$ such that $A, \imath A$, and Fix intersect transversally with $A \cap \imath A=S$ a 1-sphere and $\partial A$ having one component in $\operatorname{int}\left(l A_{0}\right)$ and the other component is $S_{2}$.

A similar statement holds if $V$ is a 3-cell, $A_{0}, A_{1}$ are 2-cells, and the $S_{i}$ are 1-cells (see Figure 3).

Proof. By transversality of Fix, by taking a sufficiently small regular neighborhood $N$ of $A_{0} \cup A_{1} \cup \imath A_{0} \cup \imath A_{1}$ we may assume one of the following holds:

(1) Fix $\cap N=\varnothing$,

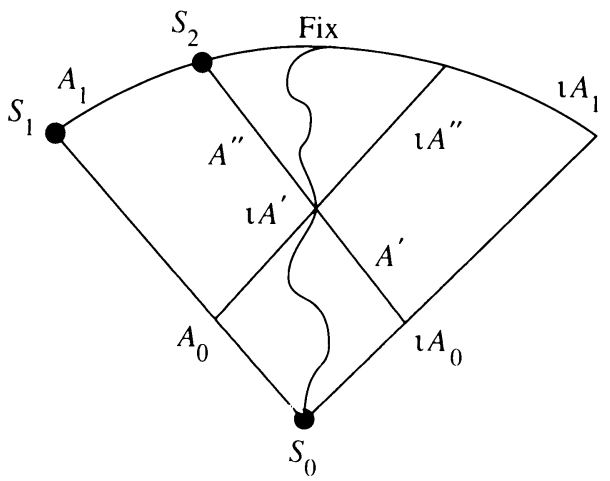

FigURE 3 
(2) Fix $\cap N$ consists of two disjoint 1-cells $I_{i}$ with exactly one point of $\partial I_{i}$ in $S_{0}$ and the other in $\operatorname{int}(V) \cap \partial N$, or (3) Fix $\cap N$ is an annulus such that one boundary component is $S_{0}$ and the other is in $\operatorname{int}(V) \cap \partial N$.

Further, there is a regular neighborhood $N^{\prime}$ of $S_{0} \subseteq N$ such that $l N^{\prime}=N^{\prime}$, $A_{0}^{\prime}=N^{\prime} \cap A_{0}$ is an annulus, $A_{0}^{\prime} \cup \imath A_{0}^{\prime}=N^{\prime} \cap \partial V$, and properties (1)-(3) hold with respect to $N^{\prime}$.

Let $B$ be the annulus which is the closure of $\partial N^{\prime}-A_{0} \cup l A_{0}$. Then there is a 1 -sphere $S$ in $\operatorname{int}(B)$ with $\imath S=S$ and Fix $\cap B \subseteq S$. There is an annulus $A^{\prime \prime}$ in $\overline{V-N^{\prime}}$ such that $\partial A^{\prime \prime}=A^{\prime \prime} \cap \overline{V-N^{\prime}}=S_{2} \cup S$ and $A^{\prime \prime} \cap \imath A^{\prime \prime}=S$. Let $A^{\prime}$ be the component of $B-S$ that meets $l A_{0}$. Then $A=A^{\prime} \cup A^{\prime \prime}$ is the desired annulus.

A solid Klein bottle is a twisted $I$-bundle over an annulus. The annulus is essential but it does not separate the boundary.

Lemma 4.2. Let $U$ be a solid torus or a solid Klein bottle. If $U$ is a solid torus then $U$ has no essential annuli. If $U$ is a solid Klein bottle then $U$ has no essential annuli that separate $\partial U$.

Moreover, suppose $A^{\prime}$ is an annulus contained in $\partial U$ such that a nonseparating proper disc $D$ of $U$ intersects $A^{\prime}$ in exactly one nonseparating 1-cell of $A^{\prime}$. If $A$ is an incompressible proper annulus disjoint from $A^{\prime}$ then the solid torus which trivializes $A$ may be taken to be disjoint from $A^{\prime}$.

Proof. Suppose $A$ is an essential annulus. Then let $D$ be any proper nonseparating 2-cell of $U$. (When $A^{\prime}$ is given, take $D$ as in the statement.) Make $A$ and $D$ transversal. Since $A$ is incompressible, adjust $D$ so that $A \cap D$ consists of 1-cells only.

If $A \cap D=\varnothing$ then $A$ is contained in a 3-cell obtained by removing a sufficiently small regular neighborhood of $D$ from $U$. This contradicts incompressibility.

If $A \cap D \neq \varnothing$ let $B$ be an outermost 2-cell of $D$ (and disjoint from $A^{\prime}$ if $A^{\prime}$ is given): so $B \cap A=\partial B \cap A=I$ is a 1-cell and $B \cap \partial U=\overline{\partial B-I}$. If $I$ bounds a 2-cell in $A$, then by an isotopy moving $B$, obtain a disc $D^{\prime}$ with fewer 1-cells in $A \cap D^{\prime}$. Assume now that $I$ does not bound a 2-cell in $A$. Then $I$ does not separate $A$. Let $V$ be the closure of the component of $U-A$ that meets $\operatorname{int}(B) . \partial A$ decomposes $\partial U$ into two annuli or possibly, in the case where $U$ is a solid Klein bottle, into an annulus and two Möbius bands. However, in the latter case $\partial B \cap \partial U$ must meet the annulus. It follows that $\partial V \cap \partial U$ is an annulus and $V$ is a solid torus with the properties making $A$ trivial.

In order to prove the torus theorem we will need to know the involutions up to conjugacy on a solid torus.

Let $S^{1}=\{z \in \complement:|z|=1\}$. Define maps on $S^{1}$ by $\kappa(z)=\bar{z}$ and $\alpha(z)=$ $-z$. Let $D^{2}=\{z \in \mathcal{C}:|z| \leq 1\}$. Define maps on $D^{2}$ by $\hat{\kappa}(z)=\bar{z}$ and 
$\hat{\alpha}(z)=-z$. Also define $\hat{\rho}$ on $D^{2} \times S^{1}$ by $\hat{\rho}(z, w)=(z w, w)$. For later use, define $\tau$ on $I=[-1,1]$ by $\tau(t)=-t$.

Define the following involutions on the solid torus $V=D^{2} \times S^{1}=\{(z, w)$ : $|z| \leq 1,|w|=1, z, w \in \complement\}$ :

$j_{A}=\hat{\kappa} \times$ id having as fixed set the annulus $\operatorname{Re} \times S^{1}$.

$j_{M}=\hat{\rho} \circ(\hat{\kappa} \times \mathrm{id})$ having as fixed set the Möbius band $\left\{\left(s \cdot e^{\pi \mathrm{i} t}, e^{2 \pi \mathrm{i} t}\right): 0 \leq\right.$ $s \leq 1,-1 \leq t \leq 1\}$.

$j_{2 D}=\mathrm{id} \times \kappa$ having as fixed set two 2-cells $D^{2} \times \pm 1$.

$j_{D P}=\hat{\rho} \circ(\mathrm{id} \times \kappa)$ having as fixed set a 2 -cell and a point $D^{2} \times 1 \cup 0 \times-1$.

$j_{S}=\hat{\alpha} \times$ id having as fixed set one 1 -sphere $0 \times S^{1}$.

$j_{2 C}=\hat{\kappa} \times \kappa$ having as fixed set two 1-cells $\operatorname{Re} \times \pm 1$.

$j_{2 P}=\hat{\alpha} \times \kappa$ having as fixed set two points $0 \times \pm 1$.

$j_{N}=\hat{\kappa} \times \alpha$, fixed point free and orientation reversing.

$j_{O}=\mathrm{id} \times \alpha$, fixed point free and orientation preserving.

The next theorem follows easily by applying the Disc Theorem 3.13 and using the fact (see [13]) that there are only three involutions up to conjugacy on a 3-cell (for details see [4]).

Theorem 4.3. If $l$ and $l^{\prime}$ are involutions on $V=D^{2} \times S^{1}$ with nonempty isomorphic fixed point sets or if $l$ and $l$ are fixed point free and of the same orientation type, then $l$ and $l^{\prime}$ are conjugate. An involution on $V$ is conjugate to one of the nine involutions listed above.

Lemma 4.4. Let $M$ be an irreducible, $P^{2}$-irreducible 3-manifold containing an incompressible torus. Let $F$ be a 1-sided Klein bottle in the interior of $M$ and $W$ a regular neighborhood of $F$ in $M$ with $\partial W$ a torus. Then $\partial W$ is an incompressible torus.

Proof. Otherwise $M=W \cup U$, where $M-W=U, \partial U=U \cap(\overline{U-W})$, and $U$ is a solid torus. Necessarily $W$ is an orientable twisted $I$-bundle over $T$ and $M$ is orientable. The inclusion of $U$ in $M$ determines an index two subgroup of $\pi_{1}(M)$. Consider $p: \widetilde{M} \rightarrow M$, the 2-sheeted covering corresponding to that subgroup. Then $p^{-1}(W)=T \times[-1,1]$, where $T$ is a torus with $p(T \times 0)=F$, and $p^{-1}(U)=V_{1} \cup V_{2}$, where $V_{1}$ and $V_{2}$ are two disjoint solid tori. $\widetilde{M}$ is a lens space. But $M$ and hence $\widetilde{M}$ contains a 2-sided incompressible torus.

Equivariant Torus Theorem 4.5. Let $M$ be an irreducible, $P^{2}$-irreducible 3manifold with involution $l$. Suppose $M$ contains an incompressible torus. Then one of the following holds:

(I) There is a 2-sided incompressible torus or Klein bottle $T$ in $\operatorname{int}(M)$ transversal to Fix with $T \cap \imath T=\varnothing$ or $\imath T=T$.

(II) $M=V_{-1} \cup V_{1} \cup U_{-1} \cup U_{1}$, where $V_{i}$ and $U_{i}$ are solid tori and $i V_{i}=V_{i}$ and ${ }_{l} U_{-1}=U_{1}$. There are annuli $A_{i}, i= \pm 1$, with

$$
A_{1} \cap A_{-1}=A_{i} \cap \imath A_{i}=\partial A_{i}=\partial \imath A_{i}=V_{1} \cap V_{-1}=U_{1} \cap U_{-1}
$$



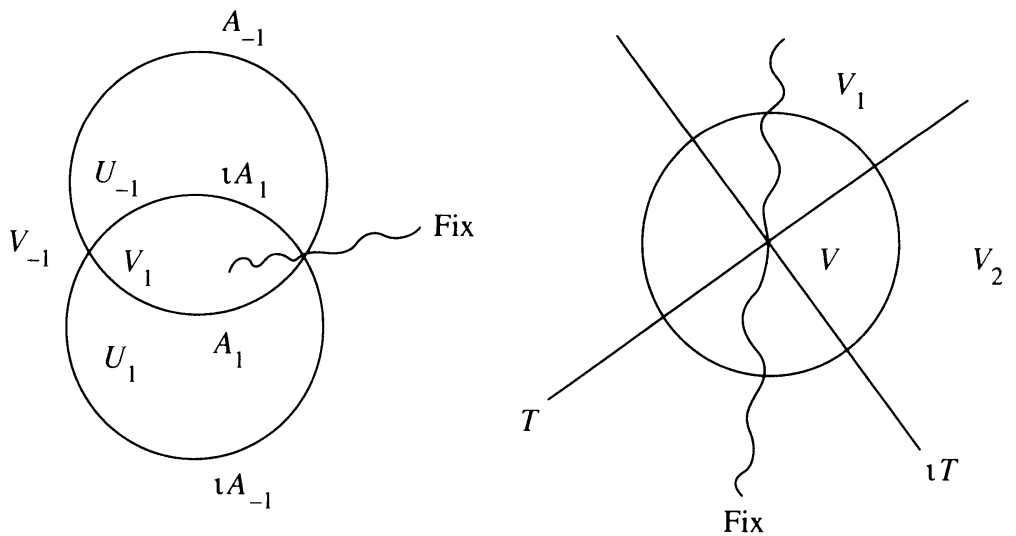

FigURE 4

and $V_{i} \cap U_{i}=A_{i}, \quad V_{i} \cap U_{-i}=l A_{i}, \quad \partial V_{i}=A_{i} \cup \imath A_{i}, \quad \partial U_{i}=A_{i} \cup \imath A_{-i}$, (see Figure 4). $A_{1} \cup A_{-1}$ is a 2-sided incompressible torus transversal to Fix. $\imath \mid V_{i}$ is orientation preserving.

(III) $M=V_{1} \cup V_{2} \cup V$, where $V_{1}, V_{2}$, and $V$ are solid tori each invariant under $l$ such that $l$ is orientation preserving when restricted to any of $V_{1}, V_{2}$, and $V$. There is a 1-sided Klein bottle $T$ with $T \cap \imath T=S \subseteq \operatorname{int}(V)$ a generator of $\pi_{1}(V) . \quad V_{1} \cap V_{2}=(T \cap \imath T)-\operatorname{int}(V)$ are two annuli. $T, \imath T$, and Fix are pairwise transversal and Fix $\cap \partial V_{2}=\varnothing$ and Fix $\cap \leqslant \neq \varnothing . V$ is a standard neighborhood of $S$, (see Figure 4).

(IV) $M=W \cup V$, where $W$ is a twisted I-bundle over a torus $T \subseteq W$ and $V$ is a solid torus with $\partial W=\partial V=W \cap V$ and $\imath W=W, \imath T=T$, and $\imath V=V$. Fix is transversal to $\partial W$ and $T$ except for a possible 1-sphere component $S$ of Fix ${ }^{1}$ contained in $T$.

Proof. Let $T_{0}$ be an incompressible torus in $\operatorname{int}(M)$. By Theorem 3.12 assume $T_{0}, l T_{0}$, and Fix are almost pairwise transversal and that no 1-spheres in $T_{0} \cap \imath T_{0}$ bound 2-cells in $T_{0}$.

As a first step we handle the cases where saddle components arise. Only Type III and Type II components are possible. In both cases since $S$ and $S_{1}$ intersect transversally at one point, there can be only one component in $T_{0} \cap \imath T_{0}$.

Suppose $T_{0} \cap \imath T_{0}$ is a Type III component $S \cup S_{1} \cup S_{2}$. Then $S_{1}$ and $S_{2}$ bound an annulus $A$ in $T_{0}$ since $S_{1} \cap S_{2}=\varnothing$ and both intersect $S$ transversally once. Then $T=A \cup l A$ is a torus with $\imath T=T$ and $T$ and Fix are transversal. $T$ is 1-sided since a regular neighborhood of $S_{1}$ is a solid Klein bottle. Let $N$ be a regular neighborhood of $T$ invariant under $N$. If $\partial N$ is incompressible then it is a 2-sided torus satisfying (I). If $\partial N$ is compressible we arrive at (IV).

Suppose $T_{0} \cap \imath T_{0}$ is a Type II component $S \cup S_{1}$. First we construct a torus $T^{\prime}$ isotopic to $T=T_{0}$ with $l T^{\prime}=T^{\prime}$. Let $N(S)$ and $N\left(S_{1}\right)$ be regular neighborhoods of $S$ and $S_{1}$ respectively, both invariant under $l$ such that $N=N(S) \cup N\left(S_{1}\right)$ is a regular neighborhood of $S \cup S_{1}$ and such that $T \cap N(S)$ 
and $T \cap N\left(S_{1}\right)$ are annuli, $N\left(S_{1}\right) \cap$ Fix is a Möbius band, $N(S) \cap$ Fix $^{2}$ is a proper 2-cell, and $N(S) \cap$ Fix $^{1}$ is a proper 1-cell. Both $N(S)$ and $N\left(S_{1}\right)$ are solid Klein bottles. By transversality there are two disjoint open 2-cell components $K_{1}$ and $K_{2}$ of $N(S)-(T \cup \imath T)$ that meet Fix ${ }^{1}$ and there are two disjoint open 2-cell components $L_{1}$ and $L_{2}$ of $N\left(S_{1}\right)-(T \cup \imath T)$ that do not meet $\mathrm{Fix}^{2}$. By considering the effect of $l$ near saddle points we see $A=\left(K_{1} \cup K_{2} \cup L_{1} \cup L_{2}\right) \cap \partial N$ is an annulus with $\partial A=C \cup \imath C$, where $C=$ $\partial N \cap T$. The closure of $A \cup(T-N) \cup l(T-N)$ is a 2-sphere which by the irreducibility of $M$ bounds a 3-cell $E$. $E$ cannot contain the proper punctured torus $T \cap N$ so $E \cap \operatorname{int}(N)=\varnothing$. Since Fix $^{1}$ is transversal to $\partial E$ and $\imath \partial E=\partial E$ it follows $\imath E=E$. In particular, $\imath \mid E$ is conjugate to $j_{1}$, the standard involution of a 3-cell with fixed set one 1-cell. $A$ is invariant and contains Fix $^{1} \cap \partial E$. Hence one shows there is a proper 2-cell $D$ with $\partial D$ a generator of $H_{1}(A)$ such that Fix $^{1} \cap E$ is a proper 1 -cell of $D$ and $\imath D=D$. Since $l \partial D=\partial D$, by taking $N$ sufficiently small we can construct a proper punctured torus $P$ in $N$ with $\partial P=\partial D$ and ${ }_{l} P=P$ (namely isotope $\left.T \cap N\right)$. Consider the torus $T^{\prime}=P \cup D$. Fix ${ }^{2}$ intersects $T^{\prime}$ transversally at $S_{1}$. The component of Fix ${ }^{1}$ meeting $N$ is contained in $T^{\prime}$. $T^{\prime}$ is 1 -sided. Let $W$ be a regular neighborhood of $T^{\prime}$ invariant under $l$. If $\partial W$ is incompressible then it is a 2-sided torus satisfying (I). If $\partial W$ is compressible we arrive at (IV).

We may now assume $T_{0} \cap \imath T_{0}$ has no saddle components. $T_{0}, \imath T_{0}$, and Fix are pairwise transversal and $T_{0} \cap \imath T_{0}$ consists of disjoint 1-spheres bounding annuli in $T_{0}$ and $l T_{0}$. We successively construct incompressible tori or Klein bottles $T$ with fewer 1 -spheres in $T \cap \imath T$, but always keep $T \cap \imath T$ consisting of 1 -spheres bounding annuli in $T$ and $l T$ and always retain the property that each component of $T \cap \imath T$ has a standard neighborhood, (see Propositions 3.9 and 3.10). It also follows then that any 1-sided Klein bottle arising from such a construction has a regular neighborhood $W$ with $\partial W$ a torus. So Lemma 4.4 is applicable.

Note: Suppose $T$ satisfies all the conditions of (I) except that $T$ is 1-sided instead of 2-sided. Let $W$ be a regular neighborhood of $T$. We can take $W$ so that $\partial W$ and Fix are transversal and $\imath W=W$ or $W \cap \imath W=\varnothing . \partial W$ is 2-sided. If $\partial W$ is incompressible, $\partial W$ satisfies (I). Assume now that $\partial W$ is compressible. $T$ cannot be a Klein bottle since the previous paragraph shows that $\partial W$ is a torus and thus Lemma 4.4 would show $\partial W$ to be incompressible. So $T$ is a torus. Now $V=\overline{M-W}$ is a solid torus. If $\imath T=T$ we have (IV). If $l T \cap T=\varnothing$ then the solid torus $V$ contains an embedded 1 -sided torus $l T$, a contradiction.

We give constructions that reduce the number of components of $T \cap \imath T$, producing a new torus or Klein bottle. Repeat these constructions with the new torus or Klein bottle produced until eventually one satisfying condition (I), (II), (III), or (IV) is constructed. There are four cases depending on the number of 1-spheres of $T \cap \imath T$ and the compressibility of certain surfaces. 

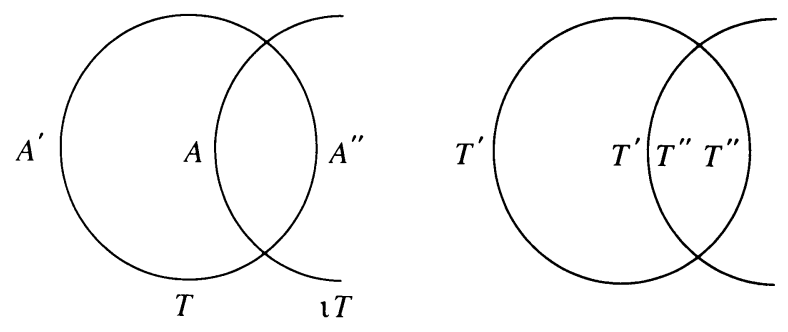

Figure 5

Assume $T \cap \imath T$ consists of at least two 1-spheres. Let $A \subseteq \imath T$ be an innermost annulus: $A \cap T=\partial A$. $\partial A$ decomposes $T$ into two annuli $A^{\prime}$ and $A^{\prime \prime}$ with $T=A^{\prime} \cup A^{\prime \prime}$ and $\partial A=\partial A^{\prime}=\partial A^{\prime \prime}=A^{\prime} \cap A^{\prime \prime} . T^{\prime}=A^{\prime} \cup A$ and $T^{\prime \prime}=A^{\prime \prime} \cup A$ are tori or Klein bottles (see Figure 5).

Case (1). $T^{\prime}$ is incompressible.

Case (1.1): $\imath \partial A=\partial A$ and $\imath A=A^{\prime}$. Then $\imath T^{\prime}=T^{\prime}$. One sees Fix is transversal to $T^{\prime}$ by considering the standard neighborhoods of $\partial A$. We arrive at (I) or (IV).

Case (1.2): Either $\imath \partial A=\partial A$ and $\imath A=A^{\prime \prime}$ or $\imath \partial A \cap \partial A$ is a single 1-sphere $S$ and $l A \subseteq A^{\prime \prime}$. In the latter case $l S=S$. Let $V_{1}$ and $V_{2}$ be distinct standard neighborhoods of $\partial A$ and let $\gamma_{1}$ and $\gamma_{2}$ be the two distinct standard annuli that meet both $A$ and $A^{\prime}$. Let $T_{1}=\left(A^{\prime} \cup A \cup \gamma_{1} \cup \gamma_{2}\right)-\operatorname{int}\left(V_{1} \cup V_{2}\right)$. Then $T_{1} \cap \imath T_{1} \subseteq(T \cap \imath T)-\partial A$ and $T_{1} \cap \imath T_{1} \neq T \cap \imath T$ because $\left(\gamma_{1} \cup \gamma_{2}\right) \cap \imath\left(\gamma_{1} \cup \gamma_{2}\right)=\varnothing$. $T^{\prime}$ and $T_{1}$ are ambient isotopic so $T_{1}$ is incompressible. Fix $\cap\left(\gamma_{1} \cup \gamma_{2}\right)=\varnothing$ and $A$ is innermost so $T_{1}, \imath T_{1}$, and Fix are pairwise transversal. Proceed with $T_{1}$.

Case (1.3): Either $\imath \partial A \cap \partial A$ is a single 1-sphere $S$ and $\imath A \subseteq A^{\prime}$ or $\imath \partial A \cap$ $\partial A=\varnothing$. Let $\partial A=S \cup S^{\prime}$. Let $V$ be a standard neighborhood of $S$ and let $\gamma$ be the standard annulus that meets both $A$ and $A^{\prime}$. Let $A \times[0, \varepsilon]$ be a sufficiently thin collar of $A=A \times 0$ in $M$ such that

$$
S^{\prime} \times[0, \varepsilon] \subseteq A^{\prime}, \quad S \times[0, \varepsilon] \subseteq T, \quad(A \cap \partial V) \times[0, \varepsilon]=(A \times[0, \varepsilon]) \cap \partial V
$$

The collar exists since $V$ is a solid torus. In the first case, $\imath S=S$ and $\imath \gamma=\gamma$. By Lemma 4.1 if $(A \times \varepsilon) \cap \gamma \neq \varnothing$ we may assume $(A \times \varepsilon) \cap V$ and $l((A \times \varepsilon) \cap V)$ intersect transversally in a 1 -sphere $S_{1}$ and that both are transversal to Fix. In all other cases set $S_{1}=S$. Define

$$
T_{1}=(A \times \varepsilon) \cup \overline{A^{\prime}-\left(\left(S^{\prime} \cup S\right) \times[0, \varepsilon]\right)} \cup\left((S \times[0, \varepsilon])-A^{\prime}\right) .
$$

Then $T_{1} \cap \imath T_{1} \subseteq((T \cap \imath T)-\partial A) \cup S_{1} . T_{1}$ is incompressible since it is ambient isotopic to $T^{\prime}$. $T_{1}, l T_{1}$, and Fix are pairwise transversal. Proceed with $T_{1}$.

By Case (1) we may now assume $T^{\prime}$ and $T^{\prime \prime}$ are compressible. 


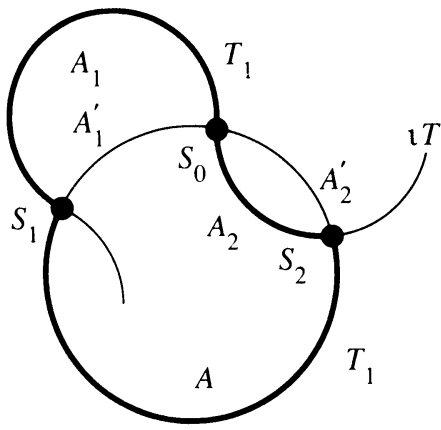

FIGURE 6

Case (2). For every annulus $A \subseteq \imath T$ with $A \cap T=\partial A$, both corresponding surfaces $T^{\prime}$ and $T^{\prime \prime}$ are compressible and $T \cap \imath T$ contains more than two 1spheres. Then let $A_{1}$ and $A_{2}$ in $\imath T$ be annuli with $A_{i} \cap T=\partial A_{i}$ and with $\partial A_{i}=S_{0} \cup S_{i}$, where $S_{0}, S_{1}$, and $S_{2}$ are 1-spheres with $S_{1} \neq S_{2}$. Let $A$, $A_{1}^{\prime}$, and $A_{2}^{\prime}$ be the three annuli of $T$ that these 1-spheres decompose $T$ into: $\partial A=S_{1} \cup S_{2}$ and $\partial A_{i}^{\prime}=S_{0} \cup S_{i}$ for $i=1,2$ (see Figure 6).

Define $T_{1}=A \cup A_{1} \cup A_{2} . T_{1}$ is incompressible. Otherwise $T_{1}$ bounds a solid torus or a Klein bottle $U$. Say $A_{1}^{\prime} \subseteq U . A_{1}^{\prime}$ is trivial in $U$ by Lemma 4.2. If $A_{1}^{\prime} \cup A_{1}$ bounds the trivializing torus then the incompressible $T=A_{1}^{\prime} \cup A_{2}^{\prime} \cup A$ is ambient isotopic to $A_{1} \cup A_{2}^{\prime} \cup A$ which was compressible by hypothesis. If $A_{1}^{\prime} \cup A_{2} \cup A$ bounds the trivializing torus then, since $A_{1}^{\prime}$ and $A_{2}$ meet on $S_{0}$, $A_{2}$ must also be trivial in $A_{1}^{\prime} \cup A_{2} \cup A$. So $T$ is ambient isotopic to $A_{2}^{\prime} \cup A_{2}$ which was assumed compressible.

We have five cases:

Case (2.1): $l\left(S_{1} \cup S_{2}\right)=S_{1} \cup S_{2}$ and $\imath S_{0} \subseteq A$. Then $l\left(A_{1} \cup A_{2}\right)=A$.

Case (2.2): $l\left(S_{1} \cup S_{2}\right)=S_{1} \cup S_{2}$ and $l S_{0} \subseteq A^{\prime}$. Then $l\left(A_{1} \cup A_{2}\right)=A_{1}^{\prime} \cup A_{2}^{\prime}$ and $\imath S_{0}=S_{0}$.

Case (2.3): $\imath S_{1}=S_{1}$ and $\imath S_{0}=S_{0}$. Then $\imath A_{1}=A_{1}^{\prime}$ and $\imath S_{2} \subseteq A_{2}^{\prime}$.

Case (2.4): $l\left(S_{1} \cup S_{2}\right) \cap\left(S_{1} \cup S_{2}\right)=\varnothing$.

Case (2.5): $l\left(S_{1} \cup S_{2}\right) \cap\left(S_{1} \cup S_{2}\right)$ is one 1-sphere.

These cases cover all possibilities. In each case we find a $T_{i}$ with fewer 1 -spheres.

Case (2.5) follows from the other cases. After relabeling assume $S_{1}$ is the 1-sphere in the intersection. Then $\imath S_{1}=S_{1}$. By Case (2.3) we assume $\imath S_{0} \neq$ $S_{0}$. Let $A_{3}$ be the innermost annulus adjacent to $A_{1}: A_{3} \subseteq \imath T_{1}$ with $A_{3} \cap$ $T_{1}=\partial A_{3}=S_{1} \cup S_{3}$, where $S_{3} \neq S_{0}$. By Case (2.3) again we may assume $l S_{3} \neq S_{3}$. By Cases (2.1) and (2.2) we may assume $\imath S_{0} \neq S_{3}$. So we have $l\left(S_{0} \cup S_{3}\right) \cap\left(S_{0} \cup S_{3}\right)=\varnothing$ and Case (2.4) gives the reduction.

In Case (2.1) use $T_{1}=\imath T_{1}$. Fix is transversal to $T_{1}$ since the standard annulus meeting $A_{1}$ and $A$ is invariant.

In Case (2.2) and Case (2.3): For $i=1,2$ let $V_{i}$ be the standard neighborhoods of $S_{i}$ with $\gamma_{i}$ the standard annuli that meet both $A$ and $A_{i}$. In all cases $\left(\gamma_{1} \cup \gamma_{2}\right) \cap l\left(\gamma_{1} \cup \gamma_{2}\right)=\varnothing$. Define $T_{2}$ to be the incompressible surface ambient 
isotopic to $T_{1}$ given by

$$
T_{2}=T_{1} \cup \gamma_{1} \cup \gamma_{2}-\operatorname{int}\left(V_{1} \cup V_{2}\right) .
$$

Then $T_{2}, \imath T_{2}$, and Fix are pairwise transversal and

$$
T_{2} \cap \imath T_{2} \subseteq T_{1} \cap \imath T_{1}-\left(S_{1} \cup S_{2}\right) .
$$

In Case (2.4): First assume $l S_{0} \neq S_{0}$. By symmetry assume $l S_{0} \neq S_{1}$. Let $\left(A_{1} \cup A_{2}\right) \times[0, \varepsilon]$ be a sufficiently thin collar of $A_{1} \cup A_{2}=\left(A_{1} \cup A_{2}\right) \times 0$ in $M$ such that $\left(S_{1} \cup S_{2}\right) \times[0, \varepsilon] \subseteq T$ and $S_{0} \times[0, \varepsilon] \subseteq A^{\prime}$. Define

$$
T_{2}=\left(A_{1} \cup A_{2}\right) \times \varepsilon \cup \overline{A-\left(\left(S_{1} \cup S_{2}\right) \times[0, \varepsilon]\right)} \cup\left(\left(S_{1} \cup S_{2}\right) \times[0, \varepsilon]-A\right) .
$$

Then $T_{2} \cap \imath T_{2} \subseteq l(T \cap \imath T)-S_{0}$. Also $T_{2}$ is ambient isotopic to incompressible $T_{1} . T_{2}, \imath T_{2}$, and Fix are pairwise transversal.

If $l S_{0}=S_{0}$, proceed as above but replace the condition $S_{0} \times[0, \varepsilon] \subseteq A^{\prime}$ by $S_{1} \times[0, \varepsilon] \subseteq A$. Use Lemma 4.1 on a standard neighborhood of $S_{0}$ to adjust the collar so that $\left(A_{1} \cup A_{2}\right) \times \varepsilon$ and $l\left(A_{1} \cup A_{2}\right) \times \varepsilon$ intersect transversally in one 1 -sphere $S_{3}$. Then $T_{2} \cap \imath T_{2} \subseteq\left(l(T \cap \imath T)-\left(S_{0} \cup S_{1}\right)\right) \cup S_{3}$.

Case (3). For each annulus $A \subseteq \imath T$ with $A \cap T=\partial A$, both corresponding surfaces $T^{\prime}$ and $T^{\prime \prime}$ are not incompressible and $T \cap_{\imath} T$ is exactly two 1-spheres. Set $\imath T=A_{-1} \cup A_{1}$ with $A_{-1} \cap A_{1}=\partial A_{1}=\partial A_{-1}=T \cap \imath T=S_{1} \cup S_{2}$. Then $T=\imath A_{-1} \cup \imath A_{1}$. There are solid tori or Klein bottles $U_{i}$ and $V_{i}$, for $i= \pm 1$, pairwise disjoint on their interiors with $\partial V_{i}=A_{i} \cup l A_{i}$ and $\partial U_{i}=A_{i} \cup l A_{-i}$. None of $U_{i}$ or $V_{i}$ are solid Klein bottles. Otherwise, if, say, $V_{1}$ is a solid Klein bottle, then since $S_{1} \cup S_{2}$ decomposes $\partial V_{1}$ into two annuli it follows that $S_{1}$ bounds a disc in $V_{1}$. This contradicts the incompressibility of $T$. By considering the standard annuli of a standard neighborhood of $S_{1}$ we see $l V_{i}=V_{i}$ and $l U_{i}=U_{-i}$. That $A_{1} \cup A_{-1}$ is a torus and not a Klein bottle follows from the fact that $V_{1}$ and $U_{-1}$ are solid tori.

If both $\imath \mid V_{1}$ and $\imath \mid V_{-1}$ are orientation preserving then we arrive at (II). So assume that $\imath \mid V_{1}$, say, is orientation reversing. We will show $l \mid V_{1}$ is conjugate to $j_{M}$ and arrive at (I). By Theorem 4.3, $\imath \mid V_{1}$ is conjugate to $j_{A}, j_{2 D}, j_{N}$, $j_{M}$, or $j_{D P}$, the standard involutions on a solid torus. $j_{2 D}$ and $j_{D P}$ are not possible since $S_{1}$ or $S_{2}$ would bound a disc contradicting the incompressibility of $T$.

If $l \mid V_{1}$ is conjugate to $j_{M}$ then, say, $S_{1} \subseteq$ Fix and $S_{2} \cap$ Fix $=\varnothing$. Then ${ }_{l} \mid V_{-1}$ has a 2-dimensional fixed set component that has only one boundary component. It follows that $l \mid V_{-1}$ is also conjugate to $j_{M}$. So Fix contains a Klein bottle $K$. There is a regular neighborhood $W$ of $K$ with $\imath \partial W=\partial W$ and $W \cap \mathrm{Fix}=\varnothing$. Since $V_{i}$ are solid tori and $K \cap V_{i}$ is a Möbius band, $\partial W$ is a torus. By Lemma 4.4, $\partial W$ is incompressible. We arrive at (I).

If $\imath \mid V_{1}$ is conjugate to $j_{A}$, then $\left[S_{1}\right]$ represents a generator of $H_{1}\left(V_{1}\right)$ and hence there is an ambient isotopy taking $l T$ to $\partial U_{-1}$ (move $A_{1}$ to $l A_{1}$ ). This contradicts that $l T$ is incompressible. 
Finally suppose $l \mid V_{1}$ is conjugate to the involution $j_{N}=\hat{\kappa} \times \alpha$ on $D^{2} \times S^{1}$. If $\imath S_{1}=S_{1}$ then $S_{1}^{\prime}=1 \times S^{1}$ and $S_{2}^{\prime}=-1 \times S^{1}$ determine annuli $A^{\prime}$ and $l A^{\prime}$ of $\partial D^{2} \times S^{1}$. It is possible to construct a conjugation $\partial V_{1} \rightarrow \partial D^{2} \times S^{1}$ taking $A_{1}$ to $A^{\prime}$. This conjugation extends to a conjugation $V_{1} \rightarrow D^{2} \times S^{1}$. But $\left[S_{1}^{\prime}\right]$ is a generator of $H_{1}\left(D^{2} \times S^{1}\right)$ and we get a contradiction as for the $j_{A}$ case above. If $l S_{1}=S_{2}$ then use $S_{1}^{\prime}=\partial D^{2} \times 1$ and $S_{2}^{\prime}=\partial D^{2} \times-1$ and proceed as above but this time obtaining a contradiction as for $j_{2 D}$ above.

Case (4). $T \cap \imath T$ is a single 1-sphere $S$. Then $\imath S=S$. Let $V$ be a standard neighborhood of $S$ and let $\alpha_{1}, \alpha_{2}, \beta_{1}$ and $\beta_{2}$ be the standard annuli with $\alpha_{1} \cap \alpha_{2}=\varnothing, \beta_{1} \cap \beta_{2}=\varnothing, l \alpha_{1}=\alpha_{1}, l \alpha_{2}=\alpha_{2}$, and $\imath \beta_{1}=\beta_{2}$. Define

$$
T_{1}=\left(T \cup \imath T \cup \alpha_{1} \cup \alpha_{2}\right)-\operatorname{int}(V)
$$

and

$$
T_{2}=\left(T \cup \imath T \cup \beta_{1} \cup \beta_{2}\right)-\operatorname{int}(V) .
$$

If $T$ is 2 -sided then $T_{1}$ is 2-sided. Also $l T_{1}=T_{1}$. Since $T$ is 2 -sided it follows that a sufficiently thin collar $T \times[0, \varepsilon]$ of $T=T \times 0$ can intersect only one of $\operatorname{int}\left(\alpha_{1}\right)$ and $\operatorname{int}\left(\alpha_{2}\right)$. Hence $T_{1}$ cannot separate and therefore $T_{1}$ is incompressible. We arrive at (I).

From now on assume $T$ is 1-sided. $T_{1}$ and $T_{2}$ are tori. This follows since $V$ is a solid torus and either both of the annuli $T-\operatorname{int}(V)$ and $l T-\operatorname{int}(V)$ are "twisted" relative to $V$ (if $T$ is a Klein bottle) or neither is (if $T$ is a torus).

If either of $T_{1}$ or $T_{2}$ is incompressible we arrive at (I). Assume then that $T_{1}$ and $T_{2}$ are compressible. Then $T_{i}$ bounds a solid torus $V_{i}$. If $S \subseteq V_{i}$ then $V_{i}$ contains a 1-sided torus or Klein bottle, a contradiction. So $M=V \cup V_{1} \cup V_{2}$ with $\operatorname{int}(V), \operatorname{int}\left(V_{1}\right)$, and $\operatorname{int}\left(V_{2}\right)$ pairwise disjoint.

By the choice of $\alpha_{1}$ and $\alpha_{2}, l$ interchanges the components of $\partial \alpha_{i}$. Therefore $l \mid \alpha_{i}$ is conjugate to one of id $\times \tau, \kappa \times \tau$, or $\alpha \times \tau$, the standard involutions of $S^{1} \times I$, where $I=[-1,1]$ and $\tau(t)=-t$. Let $S_{i}$ be a 1 -sphere of $\alpha_{i}$ that is the image of $S^{1} \times 0$ under some conjugation. Note that $S_{i}$ does not bound a disc $D$ in $V_{1}$, otherwise $T$ would be compressible.

If there is an annulus $A \subseteq V_{1}$ with $\partial A=S_{1} \cup S_{2}$ and $l A=A$ then we arrive at (IV) as follows. Torus $V_{1}$ is separated by $A$. Since $l$ interchanges the components of $\partial \alpha_{1}, l$ interchanges the components of $A-V_{1} . A$ is trivial in $V_{1}$ so it follows that $V_{1}$ can be given a trivial $I$-bundle structure over $A$. There is an annulus $B \subseteq V$ with $\partial B=S_{1} \cup S_{2}$ and $l B=B . V$ is an $I$-bundle over $B$. Consider $T_{3}=A \cup B$. It follows that $V \cup V_{1}$ is an $I$-bundle over $T_{3}$ with $\partial\left(V \cup V_{1}\right)=T_{2}$ a torus. Moreover $T_{3}$ does not separate $T$ so $T_{3}$ is 1-sided. Since $T_{2}$ is compressible, $T_{3}$ is not a Klein bottle in view of Lemma 4.4. Thus $T_{3}$ is a torus and we arrive at (IV).

Assume now that such an annulus $A$ does not exist. Since $V$ is a standard neighborhood, Fix $\cap \alpha_{1}=\varnothing$ if and only if Fix $\cap \alpha_{2}=\varnothing$. Therefore $l \mid \alpha_{1}$ and $\imath \mid \alpha_{2}$ are conjugate. 
Case (4.1): $l \mid \alpha_{1}$ is conjugate to id $\times \tau$. Then $\imath \mid V_{1}$ has a 2-dimensional fixed set that meets $\partial V_{1}$ in two fixed 1-spheres. It follows that $S_{1}$ bounds a disc or $S_{1} \cup S_{2}$ bounds an annulus $A$ fixed by $l \mid V_{1}$. By the above comments, we arrive at (IV).

Case (4.2): $\imath \mid \alpha_{1}$ is conjugate to $\alpha \times \tau$. Then $\imath \mid V_{1}$ is orientation reversing. $\imath \mid \partial V_{1}$ is conjugate to $\alpha \times \kappa$ on $S^{1} \times S^{1}$ by a conjugation taking $S_{i}$ to $S^{1} \times(-1)^{i}$. Then $l \mid V_{1}$ is conjugate to $\hat{\alpha} \times \kappa$ or $\alpha \times \hat{\kappa}$ by a conjugation extending the one given on the boundaries. In the first case $S_{1}$ bounds a disc and in the second case $S_{1} \cup S_{2}$ bounds an annulus with $l A=A$. Again by the above comments we arrive at (IV).

Case (4.3): $l \mid \alpha_{1}$ is conjugate to $\kappa \times \tau$. Then $l \mid V_{1}$ and $\imath \mid V$ are orientation preserving. Now $l \mid V_{2}$ is orientation reversing if and only if $T$ is a torus. To see this let $S_{1}=\alpha_{1} \cap \beta_{1}$ and without loss say $S_{1} \subseteq T$. Orient $S_{1} . S_{1}$ and $\imath S_{1}$ bound two annuli $A_{1}$ and $A_{2}$ of $\partial V_{2}$ with $\imath A_{1}=A_{2}$. Consider the ways of inducing an orientation on $l S_{1}$. The orientation induced by $A_{1}$ and the orientation induced by $\alpha_{1}$ are the same if and only if $T$ is a torus. Since $l \mid \alpha_{1}$ is orientation reversing the orientation induced by $\alpha_{1}$ and the orientation induced by $l$ are opposite. So $l$ and $A_{1}$ induce opposite orientations on $l S_{1}$ if and only if $T$ is a torus. Since $l A_{1}=A_{2}$ the claim follows.

If $T$ is a torus then $l \mid V_{2}$ is orientation reversing, so $l \mid \partial V_{2}$ is conjugate to the involution $\alpha \times \kappa$ on $S^{1} \times S^{1}$ by a conjugation taking $S_{1}$ to $\mathrm{i} \times S^{1}$. As in Case (4.2) we arrive at (I) or (IV).

If $T$ is a Klein bottle then we arrive at (III). $l \mid \partial V_{2}$ is fixed point free so $l \mid V_{2}$ is conjugate to $j_{S}$ or $j_{O}$ while $\imath \mid V_{1}$ is conjugate to $j_{2 C}$.

Corollary 4.6. Let $M$ be an irreducible, $P^{2}$-irreducible 3-manifold with involution 1 . Suppose $M$ contains an incompressible torus. Suppose $M$ is neither an orientable Seifert fiber space over the 2-sphere with four exceptional fibers nor a nonorientable Seifert fiber space over the projective plane with at most one exceptional fiber. Then there is a 2-sided incompressible torus or Klein bottle $T$ in $\operatorname{int}(M)$ transversal to Fix with $T \cap \imath T=\varnothing$ or $\imath T=T$.

Proof. Case (II) in the Equivariant Torus Theorem can only occur if $M$ is an orientable Seifert fiber space over the 2-sphere with four exceptional fibers, while Cases (III) and (IV) can only occur if $M$ is a nonorientable Seifert fiber space over the projective plane with at most one exceptional fiber. Since these possibilities have been excluded, only Case (I) of the Equivariant Torus Theorem remains.

\section{REFERENCES}

1. A. L. Edmonds, $A$ topological proof of the equivariant Dehn lemma, Trans. Amer. Math. Soc. 297 (1986), 605-615.

2. C. McA. Gordon and R. A. Litherland, Incompressible surfaces in branched coverings, The Smith Conjecture, Pure and Appl. Math, vol. 112, Academic Press, 1984, pp. 139-152. 
3. J. Hempel, 3-manifolds, Ann. of Math. Stud., no. 86, Princeton Univ. Press, Princeton, N.J., 1976.

4. W. H. Holzmann, Involutions with 1-or 2-dimensional fixed point sets on orientable torus bundles over a 1-sphere and on unions of orientable twisted I-bundles over a Klein bottle, Ph.D. thesis, University of British Columbia, Vancouver, B. C., 1984.

5. P. K. Kim and J. Tollefson, $P L$ involutions of fibered 3-manifolds, Trans. Amer. Math. Soc. 232 (1977), 221-237.

6. $\ldots$ Splitting the PL involutions of nonprime 3-manifolds, Michigan Math. J. 27 (1980), 259-274.

7. K. Kwun and J. Tollefson, $P L$ involutions of $S^{1} \times S^{1} \times S^{1}$, Trans. Amer. Math. Soc. 203 (1975), 97-106.

8. E. Luft, Equivariant surgery on incompressible tori and Klein bottles in 3-manifolds with respect to involutions, Math. Ann. 272 (1985), 519-544.

9. E. Luft and D. Sjerve, Involutions with isolated fixed points on orientable flat 3-dimensional space forms, Trans. Amer. Math. Soc. 285 (1984), 305-336.

10. W. H. Meeks and S. T. Yau, The equivariant Dehn's lemma and loop theorem, Comment. Math. Helv. 56 (1981), 225-239.

11. P. Scott, A new proof of the annulus and torus theorems, Amer. J. Math 102 (1980), 241-277.

12. J. Tollefson, Involutions of sufficiently large 3-manifolds, Topology 20 (1981), 323-352.

13. F. Waldhausen, Über Involutionen der 3-Sphäre, Topology 8 (1969), 81-91.

Department of Mathematics and Computer Science, University of Lethbridge, 4401 University Drive, Lethbridge, Alberta, T1K 3M4 CaNada 